This item was submitted to Loughborough's Research Repository by the author.

Items in Figshare are protected by copyright, with all rights reserved, unless otherwise indicated.

\title{
Optically reconfigurable balanced dipole antenna
}

PLEASE CITE THE PUBLISHED VERSION

PUBLISHER

(C) Institution of Electrical Engineers

LICENCE

CC BY-NC-ND 4.0

REPOSITORY RECORD

Panagamuwa, C.J., and J.C. Vardaxoglou. 2019. "Optically Reconfigurable Balanced Dipole Antenna". figshare. https://hdl.handle.net/2134/2966. 
This item was submitted to Loughborough's Institutional Repository by the author and is made available under the following Creative Commons Licence conditions.

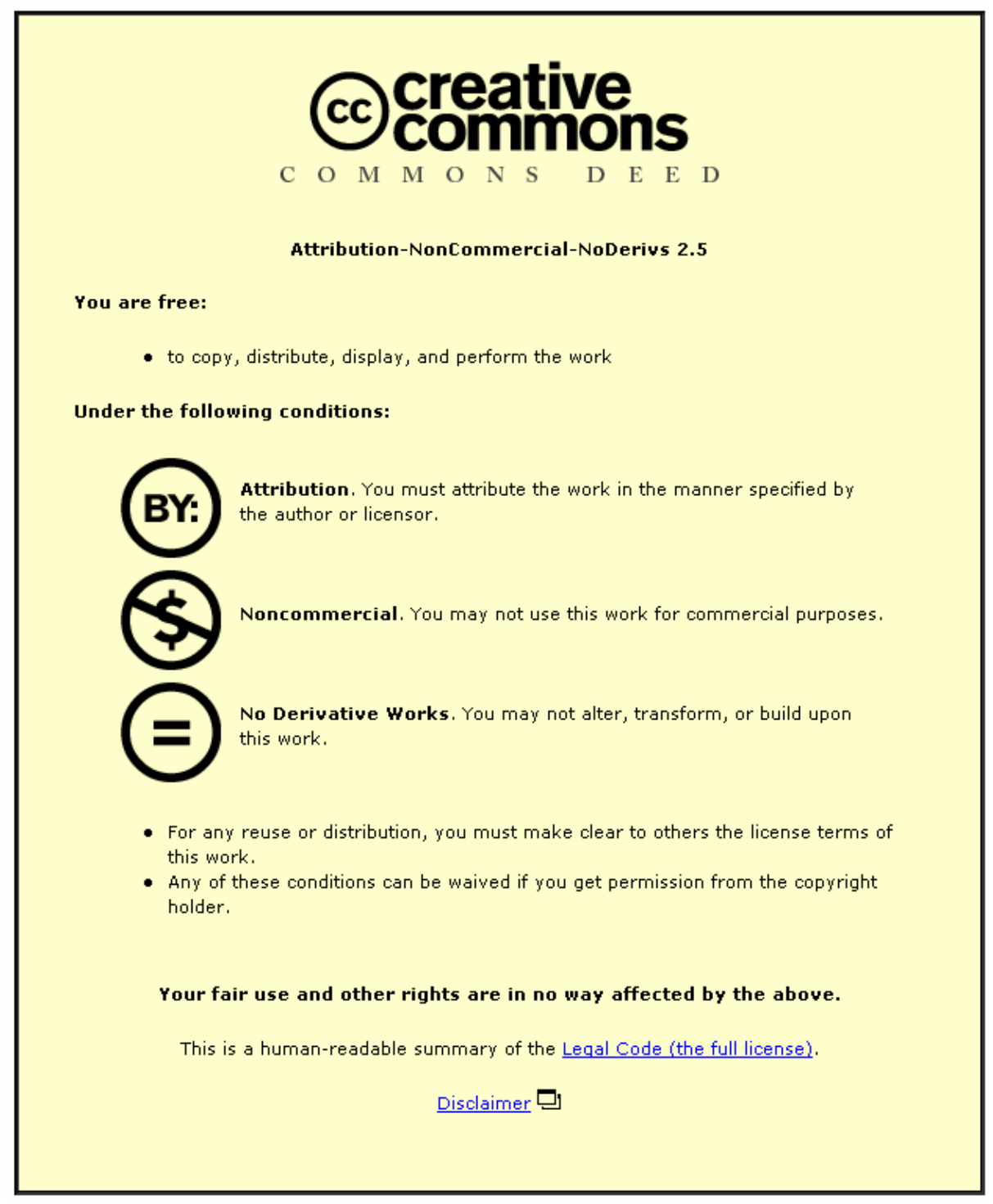

For the full text of this licence, please go to: http://creativecommons.org/licenses/by-nc-nd/2.5/ 


\title{
OPTICALLY RECONFIGURABLE BALANCED DIPOLE ANTENNA
}

\author{
C J Panagamuwa, J C Vardaxoglou
}

Loughborough University, UK

\begin{abstract}
In this paper, a new design for an optically reconfigurable printed dipole antenna is presented. A wideband coplanar waveguide $(\mathrm{CPW})$ to coplanar strip (CPS) transition is used to feed the printed dipole. Two optically activated silicon switches, controlled using fibre optic cables and near infra-red laser diodes, are placed on small gaps in the dipole arms. The switches enable the dipole length to be optically controlled, thus facilitating frequency switching. Measured return loss results that compare well to the simulated values are also presented, showing a frequency shift of $10.5 \%$.
\end{abstract}

\section{INTRODUCTION}

With the increase in demand for multiband antennas in recent years, reconfigurable active antennas have become an attractive option. A frequency switchable antenna can switch from one frequency to another, eliminating the filters often needed in wideband antenna solutions.

CPW and CPS have many advantages in RF circuit design. For example, they allow the easy placement of shunt and series passive and active devices, and have no need for via-holes to connect to ground plane. Tilley et al (1) presented a CPW-fed CPS dipole antenna with a wideband balun, which was later modified by Kolsrud et al (2) to include varactor diodes for frequency tuning. Many designs have been proposed in the past that use the variable reactance property of varactor diodes, but these are normally accompanied by biasing lines and high biasing voltages $(3,4)$. PIN diodes in reconfigurable antennas have also gained in popularity, as they require lower biasing voltages (5). These designs still require biasing lines to be attached to the antenna. As the fibre optic cables have no metallic elements, they have the advantage of being electromagnetically transparent and so do not interfere with the radiation patterns of the antenna.

The switches in the antenna are made of high resistivity silicon $(\rho>6000 \Omega \mathrm{cm})$. The semiconductor properties of silicon can be varied by photoexcitation in such a way that when illuminated by light, silicon will change from an insulator state to a near conducting state. The incident photons must have enough energy to displace the free electrons in the silicon from the valence band to the conduction band. The density of the generated plasma, the wavelength of the light and the carrier lifetimes are important considerations. The absorption coefficient and the light penetration depth are inversely proportional to each other and are related to the wavelength of the light. To achieve maximum plasma density for a given optical power, it is important to strike a balance between the absorption coefficient and light penetration depth by choosing an appropriate wavelength. At $980 \mathrm{~nm}$, a penetration depth of about $200 \mu \mathrm{m}$ is achieved. Light delivery on silicon wafers for generation and switching of frequency selective surface (FSS) arrays has been extensively studied by Vardaxoglou $(6,7,8)$. Here, we adapt a similar model regarding the properties of silicon under optical illumination perpendicular to the direction of travel of the microwave signal.

\section{DIPOLE ANTENNA}

The schematic of the dipole antenna is shown in Fig. 1. It is printed on $1.17 \mathrm{~mm}$ thick TLY $-5^{\circledR}$ substrate that has a dielectric constant $\left(\varepsilon_{\mathrm{r}}\right)$ of 2.2. There is no ground plane on the underside of the printed dipole, allowing the antenna to radiate above and below the substrate. The antenna is fed at the CPW using a 50 $\Omega$ SMA connector.

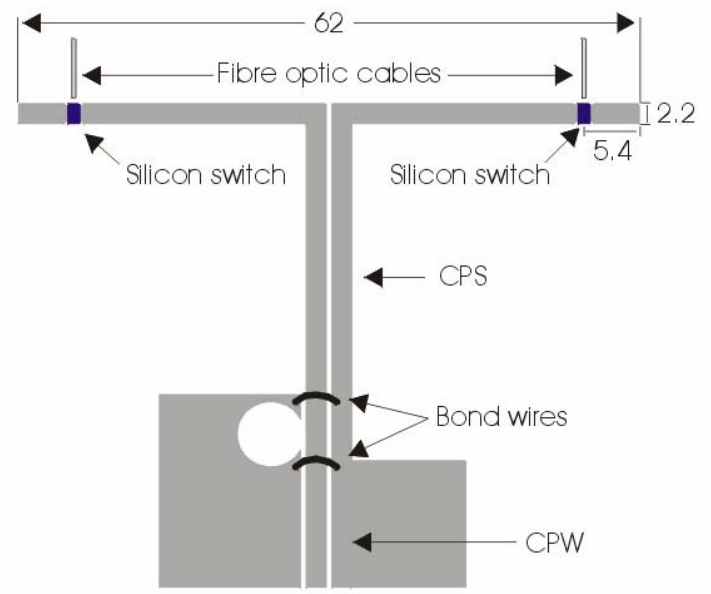

Fig. 1 - Optically reconfigurable CPS dipole antenna (dimensions given in millimetres)

The CPW is an unbalanced transmission line but since the dipole is a balanced antenna, its feed has to be 
balanced. The circular balun is used to convert the unbalanced CPW to a balanced CPS. The empty circle is a stub that has a non uniform impedance. This has a higher bandwidth compared to a uniform impedance stub. It forces the electric field to be concentrated between the two strip lines. While current on the CPW centre strip continues to flow along the left strip line, the backward current on the other strip line crosses over to the CPW ground on the right through the two bond wires, making both ground planes equal in potential. In this way, the CPW mode is effectively transformed to a CPS mode. As the CPW ground planes are of finite width and the circle introduces discontinuities, the parasitic odd mode can be excited. The use of bond wires, prevents unnecessary modes from exciting by shorting them out. Simulations of two back-to-back transitions show an insertion loss better than $1 \mathrm{~dB}$ from 1 to $3 \mathrm{GHz}$, which is suitable for the switching range of the dipole antenna.

The dipole resonates when its length is equal to $\lambda_{\text {eff }} / 2$ where $\lambda_{\text {eff }}$ is the effective wavelength. $\lambda_{\text {eff }}$ is given by $\lambda_{0} / \sqrt{ } \varepsilon_{\text {eff }}$ where $\lambda_{0}$ is the wavelength in free-space and $\varepsilon_{\text {eff }}$ is the effective dielectric constant. Although the effects of dielectric loading cannot be ignored, the absence of a ground plane under the antenna reduces the value of $\varepsilon_{\text {eff }}$ close to 1.2 . $2 \mathrm{~mm} \times 1 \mathrm{~mm} \times 0.3 \mathrm{~mm}$ silicon slabs are placed over small gaps in the dipole arms. Increasing or decreasing the length of the dipole by optically activating these silicon switches changes the resonant length, thus achieves frequency shifting. Two 980nm lasers operating at $100 \mathrm{~mW}$ are used to illuminate the switches. When both switches are turned on, the silicon starts to conduct and the dipole arms increase in length, and hence, the resonance frequency is lowered. In the OFF state, silicon behaves much like an insulator, making the dipole arms shorter. So it resonates at a higher frequency. Work previously carried out on switching microstrip transmission lines by Chauraya et al (9) showed by designing a suitable capacitance across the gap, an isolation in the OFF state of typically $15 \mathrm{~dB}$ at $2.4 \mathrm{GHz}$ could be achieved. The silicon slabs are held in place using silver loaded epoxy, which ensures good contact between the copper and silicon. The antenna design was first optimised using Microwave Office and then Micro-Stripes.

\section{RESULTS}

Measured return loss results compare well with those obtained from simulations in Micro-Stripes and are shown in Fig. 2

In addition to these standard modes of operation, each side of the dipole was switched on individually and measurements taken. These two modes of operation were also simulated in Micro-Stripes.

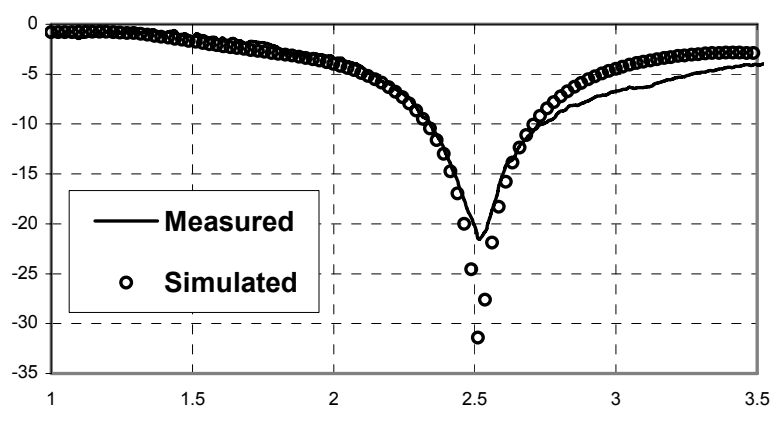

(a)

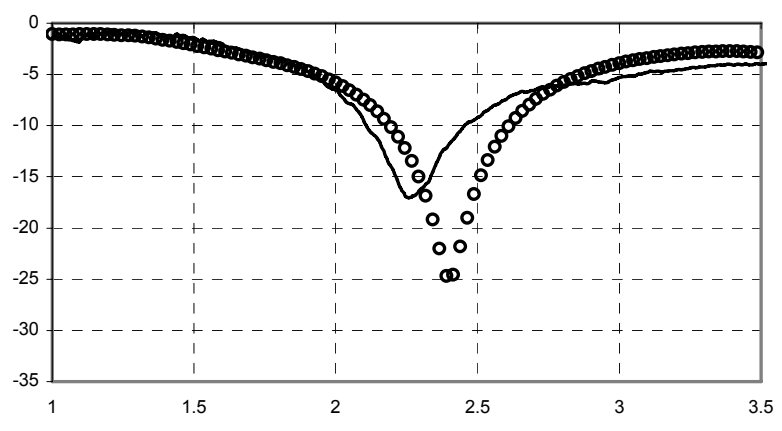

(b)

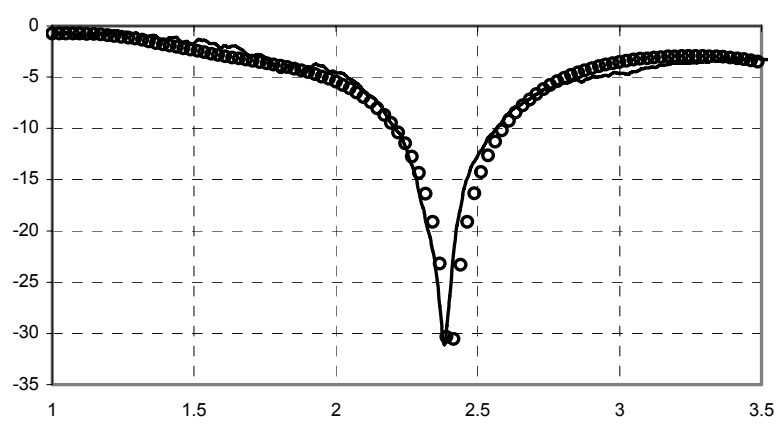

(c)

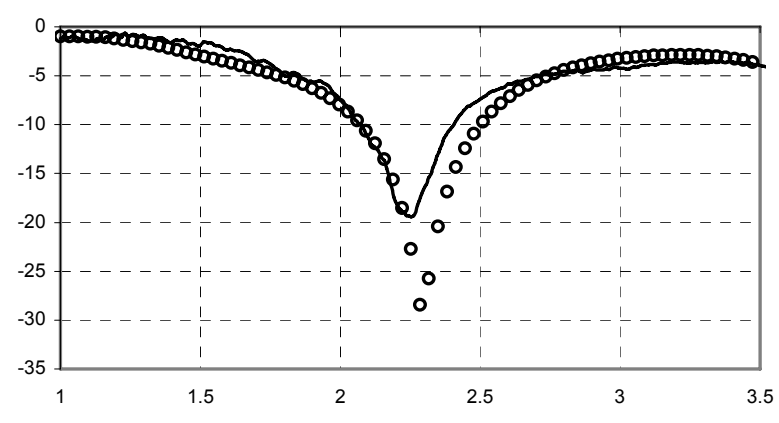

(d)

Fig. 2 - Measured and simulated return loss for dipole antenna in the following operating states (From the top down): (a) Both OFF (b) Right ON (c) Left ON (d) Both $\mathrm{ON}$ 
Two extra printed dipoles without silicon switches were built for comparison with the reconfigurable antenna. The first antenna (62 $\mathrm{mm}$ long with no gaps) represented the ideal ON state where the switch would form a perfect path over the gap with no insertion losses. The resonant frequency of this antenna was only $20 \mathrm{MHz}$ lower than the reconfigurable antenna in the ON state. The work carried out previously on transmission lines (9) showed a switch insertion loss better than $-1 \mathrm{~dB}$ in the ON state. The very close frequency mach between the two antennas shows that the switch will operate just as well in an antenna configuration.

The second antenna (51.2mm long with no gaps) represented the ideal OFF state where perfect isolation would exist between elements on either side of the gap. The shorter length antenna was found to resonate $120 \mathrm{MHz}$ higher than the reconfigurable antenna in the OFF state. However, comparing the short antenna with the reconfigurable one before the silicon slabs were mounted in place, they were found to resonate at exactly the same frequency. This shows that there is very little coupling in the tapered gap but it increases when the silicon is fixed in place. In the OFF state, the impedance of the silicon is dominated by the very high reactance. Adding this reactance to the gaps in the dipole causes the electrical length to increase, therefore causing the printed dipole to resonate at a lower frequency.

From both switches ON to both switches OFF, the resonant frequency shifts from $2.251 \mathrm{GHz}$ to $2.517 \mathrm{GHz}$. This is a shift of $10.5 \%$. When only the right switch is ON the antenna resonates at $2.381 \mathrm{GHz}$ and with just the left switch ON, it resonates at $2.331 \mathrm{GHz}$. These are frequency shifts of $5.4 \%$ and $7.3 \%$ respectively from the OFF state. The $-15 \mathrm{~dB}$ bandwidth is $6.8 \%$ for both $\mathrm{ON}$ and both OFF states. For right switch $\mathrm{ON}$ and left switch $\mathrm{ON}$, the $-15 \mathrm{~dB}$ bandwidths are $7.5 \%$ and $5.3 \%$ respectively.

The E and H-plane radiation patterns for all four operational states are shown in Fig. 3. The patterns conform well to the typical figure-of-eight patterns expected from a standard half wavelength dipole and they all have a very wide beam width. The small ripple on the patterns are due to the measurement chamber limitations. The plasma generated in a silicon switch is lossy and so it is expected that the received power when both switches are ON will be lower than that in the OFF state. However, the measurements showed no noticeable change in received power. This could be explained by the change in antenna gain with frequency for both receiver and transmitter antennas. Comparing both switches ON with both switches OFF, the E-plane pattern shapes are very similar indicating that the activation of the switches are not having a detrimental effect on the radiation patterns.
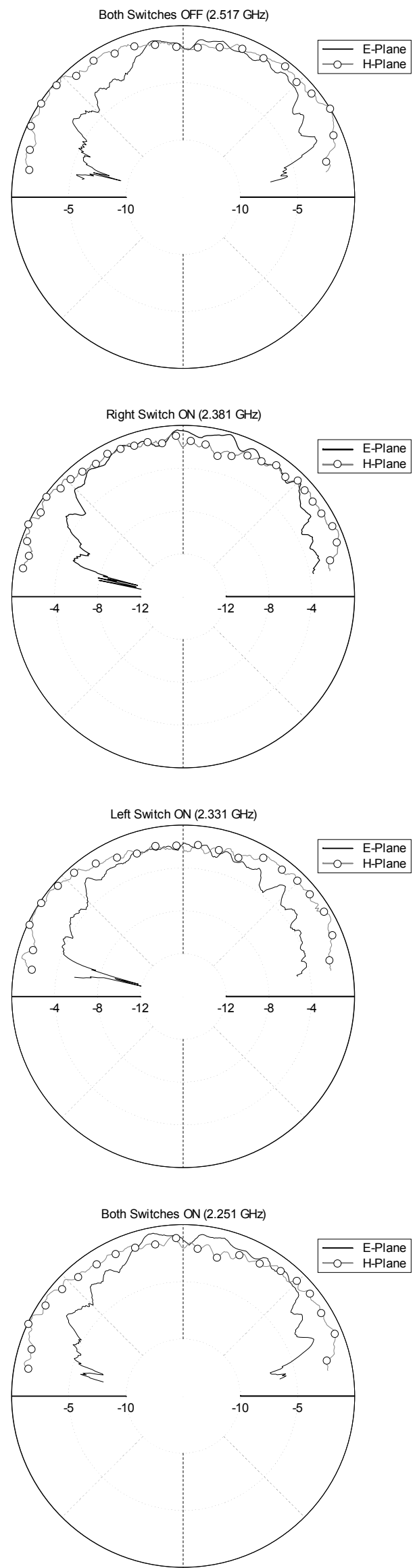

Fig. 3 - E-Plane and H-Plane radiation patterns for the printed antenna in all four operating states 


\section{CONCLUSION}

A new optically reconfigurable CPW-fed CPS dipole antenna has been successfully designed and tested. A frequency shift of $10.5 \%$ is achieved from both switches OFF to both switches ON, while preserving the $-15 \mathrm{~dB}$ bandwidth at $6.8 \%$. E and $\mathrm{H}$-field radiation patterns show no adverse effects caused by the fiber optic cables.

\section{REFERENCES}

1. Tilly K, Wu X-D and Chang K, 1994, "Coplanar waveguide fed coplanar strip dipole antenna", Electron. Lett., $\underline{30}, 176-177$

2. Kolsrud A T, Li M -Y and Chang K, 1998, "Dualfrequency electronically tunable CPW-fed CPS dipole antenna", Electron. Lett., $\underline{34}$, 609-611

3. Carrère J M, Staraj R and Kossiavas G, 2001, "Small frequency agile antennas", Electron. Lett., 37, 728-729

4. Turki Y and Staraj R, 2000, "CPW-fed frequencyagile shorted patch”, Microw. Opt. Technol. Lett., 25, 291-294

5. Luxey C, Dussopt L, Le Sonn J - L and Laheurte J M, 2000, "Dual-frequency operation of CPW-fed antenna controlled by pin diodes", Electron. Lett., $\underline{36}$, 23

6. Vardaxoglou J C, 1996, "Optical switching of frequency selective surface bandpass response", Electron. Lett., $\underline{32}$, 2345-2346

7. Vardaxoglou J C, 1997, “Optical switching and generation of periodic arrays and FSS", Int. Conf. Electromagnetics in Advanced Apps., 51-56

8. Lockyer D S, Vardaxoglou J C and Kearney M J, 1999, "Transmission through optically generated inductive grid arrays", IEEE Trans. Microwave theory Tech., 47, 1391-1397

9. Chauraya A, Lockyer D L, Lee Y L R and Vardaxoglou J C, 2001, “A study of optically tuned metallodielectric photonic band gap array and patch antenna", $11^{\text {th }}$ Int. Conf. Antennas Propagation, 492-496 